\title{
Damage Detection and Characterization in Smart Material Structures * $\dagger$
}

\author{
H.T. Banks and Y. Wang \\ Center for Research in Scientific Computation \\ North Carolina State University \\ Raleigh, NC 27695-8205
}

November, 1993

*Invited Lecture, International Conference on Control and Estimation of Distributed Parameter Systems, Vorau, Austria, July 18-24, 1993.

${ }^{\dagger}$ Research supported in part by the Air Force Office of Scientific Research under grants AFOSR F49620-93-1-0198 and AFOSR F49620-93-1-0280. Part of this research was carried out while both authors were visiting scientists at the Institute for Computer Application in Science and Engineering (ICASE), NASA Langly Research Center, which is supported by NASA under Contract Nos. NAS118605 and NAS1-19480. 


\section{Introduction}

It has been known for some time that damage such as cracks, corrosions, and delaminations in a structure produces changes in mass, stiffness, damping and other characteristics and material parameters in dynamic response models for the structure. Our focus in this paper is the development of vibration response ideas for piezoceramic based smart material structures in which self-testing nondestructive evaluation (NDE) techniques may be employed. There are several ways to formulate and analyze concepts and questions related to this quest. One could use the co-called "methodof-maps" as done in the thermal based tomography techniques of [BK1, BK2, BKW] wherein the damaged physical domain (the damaged beam, plate, elastic structure) is mapped into a regular domain on which dynamic equations with damage dependent coefficients must hold. An alternative technique involves direct estimation of the irregular damaged domain (i.e., structures with holes, cracks, corrosions, etc.) on which the usual dynamic equations hold.

The approach we take here is, strictly speaking, neither of the above, but it is more in the spirit of the latter one; we attempt to estimate damage-related changes in actual physical parameters (stiffness, damping) based on changes in physical geometry and characteristics.

Most of the previous efforts in the substantial literature on vibration related damage detection are based on modal methods (e.g. [ABB, AC, ACPS, CR, IC, $\mathrm{S}]$ ). The basis for such methods is that damage produces a decrease in dynamic stiffness EI. This decrease in turn produces decreases in natural frequencies for an undamped simple beam (recall the eigenvalues are given by $\lambda \sim \sqrt{E I / \rho A}$ where $A$ is the cross sectional area of the beam and $\rho$ is the mass density). While modal based methods may have certain advantages (e.g., they are simple if they do work, they can be used in development of rank-ordering of fractional eigenfrequency shift schemes that are reportedly insensitive to the magnitude of the damage[ABB]), modal based methods possess a number of major disadvantages. First of all, some of the modal based method investigations (e.g. [CR]) provide a strong argument for including geometry of the damage in any NDE testing scheme, something which is not easily done in frequency based methods. Indeed, mode and frequency charaterizations are not so simple in variable structure systems; there is ample evidence (e.g. see $[\mathrm{S}]$ ) that one should not use modal methods based on uniform undamped simple beams or plates as is often done in the engineering literature in addressing damage assessment methodologies. Since material parameters are most properly considered as spatially dependent quantities with damage manifested by changes in geometry (and hence in 
the spatial dependence of these parameters), it is unlikely that any rigorous theoretical basis for modal based methods for variable material structures will emerge. But perhaps the most serious objection to modal based methods resides in the fact that modal based methods have been shown to be highly unreliable (indeed inadequate! - see $[\mathrm{BI}]$ and the references therein) for estimation of variable material parameters such as damping in composite material structures.

In light of the above comments, a question of rather great interest then is: can one develop analytically sound, non-modal based self-excitation/self-sensing methods for detection and characterization (geometrical and quantitative) of damage in smart material structures? Here we address this question in the context of embedded piezoceramic structures.

For an embedded piezoceramics smart material damage detection and characterization methodology, there are several distinct requirements. These include:

(a) One must be able to estimate reliably (repeatable across experiments) the variable structure material parameters of a piezoceramic loaded structure. This must be done using piezo actuation and sensing with accuracy comparable to that achievable with standard actuating (impulse hammers, solenoidal actuators) and sensing (accelerometers, strain gauges, laser vibrometers) devices in non-smart material testing schemes.

(b) One must be able to use the actuation and sensing properties of the piezoceramics to excite the structure and analyze the response (in a single experiment) for a reliable methodology that is the basis of self-excitation/self-sensing.

(c) One must be able to detect and characterize damage via vibration self-excitation/ self-sensing that relies only on the input/output signals for the piezoceramics.

In this paper we address each of these requirements in the context of a piezoceramic loaded beam. This particular structure is sufficiently representative to make a compelling case for feasibility of the ideas we propose. In Section 2 below, we summarize a rigorous theoretical well-posedness and approximation foundation for the distributed parameter identification methodology that is the focus of our recent efforts. This is followed by Section 3 in which we detail results from experiments and computations that provide a favorable comparison for piezo actuating/sensing with standard methods (requirement (a) above). We then present experimental/computational findings on self-excitation/self-sensing capabilities of piezoceramic loaded beams. These findings suggest that requirement (b) above can indeed be satisfied.

Finally, in Section 4 we offer preliminary simulation findings on the use of vibration experiments to detect and characterize (geometrically) the extent of several 
types of damage in a beam using piezo actuation and sensing. Experimental verification of these positive findings with regard to requirement (c) is currently underway.

\section{Distributed parameter identification methodology}

The system we consider here is a cantilevered beam with piezoelectric ceramic patches for actuation and sensing. Our choice of structure is motivated by its simplicity and its representative nature. This configuration has also been well studied by conventional approaches such as finite element methods and provides a standard test bed model for comparison. The structure and model reveal the difficulties and possibilities inherent in developing models and methods for more complex structures containing damages.

We consider a cantilevered Euler-Bernoulli beam of length $\ell$ fixed at $x=0$ and free at $x=\ell$. The transverse vibrations $y=y(t, x)$ are described by the system

$$
\begin{aligned}
& \rho(x) \frac{\partial^{2} y}{\partial t^{2}}(t, x)+\gamma \frac{\partial y}{\partial t}(t, x)+\frac{\partial^{2} M}{\partial x^{2}}(t, x)=\tilde{f}(t, x) \quad 0<x<\ell, t>0 \\
& y(t, 0)=\frac{\partial y}{\partial x}(t, 0)=0, \quad M(t, \ell)=\frac{\partial M}{\partial x}(t, \ell)=0
\end{aligned}
$$

where $\rho(x)$ is the linear mass density, $\gamma$ is the coefficient of viscous (air) damping, $M(t, x)$ is the internal moment and $\tilde{f}(t, x)$ represents the external loads. For a simple Euler-Bernoulli beam with Kelvin-Voigt or strain rate damping, the internal moment is composed of two components representing resistance to bending (with coefficient $E I(x))$ and damping (with coefficient $\left.c_{D} I(x)\right)$ :

$$
M(t, x)=E I(x) \frac{\partial^{2} y}{\partial x^{2}}(t, x)+c_{D} I(x) \frac{\partial^{3} y}{\partial x^{2} \partial t}(t, x)
$$

If piezoelectric elements are bonded to the beam in a configuration to produce (or sense) only bending, we have an actuator contribution $M_{p}(t, x)$ in the form of an input moment (or voltage output proportional to the accumulated strain in the element). For a pair of piezoelectric actuators located between $x_{1}$ and $x_{2}$ on opposite sides of the beam excited by a voltage $u(t)$ in an out-of-phase manner (see [BSW, CA, CFW, DFR, FC]), this moment term has the representation

$$
M_{p}(t, x)=K_{B}\left\{H\left(x-x_{1}\right)-H\left(x-x_{2}\right)\right\} u(t)
$$

where $H(x)$ is the Heaviside or unit step function and $K_{B}$ is a piezoelectric material parameter depending on the material piezoelectric properties as well as geometry. When the moment in (3) is added to that of (2) and substituted into (1), we obtain the model 


$$
\begin{gathered}
\rho \frac{\partial^{2} y}{\partial t^{2}}+\gamma \frac{\partial y}{\partial t}+\frac{\partial^{2}}{\partial x^{2}}\left(E I \frac{\partial^{2} y}{\partial x^{2}}+c_{D} I \frac{\partial^{3} y}{\partial x^{2} \partial t}\right) \\
=K_{B}\left(\frac{d}{d x} \delta\left(x-x_{2}\right)-\frac{d}{d x} \delta\left(x-x_{1}\right)\right) u(t)+\tilde{f}(t, x) \\
y(t, 0)=\frac{\partial y}{\partial x}(t, 0)=0, \quad M(t, \ell)=\frac{\partial M}{\partial x}(t, \ell)=0
\end{gathered}
$$

where $\delta$ is the Dirac delta function. This is formally equivalent to the equation in weak or variational form (we replace partial derivatives in time by subscript $t$ and space by superscript ')

$$
\begin{aligned}
& \left\langle\rho y_{t t}+\gamma y_{t}, \phi\right\rangle+\left\langle E I y^{\prime \prime}+c_{D} I y_{t}^{\prime \prime}+K_{B}\left(H_{1}-H_{2}\right) u(t), \phi^{\prime \prime}\right\rangle=\langle\tilde{f}(t, x), \phi\rangle \\
& y(t, 0)=y^{\prime}(t, 0)=0,
\end{aligned}
$$

for sufficiently smooth functions $\phi$ satisfying $\phi(0)=\phi^{\prime}(0)=0$. Here $H_{i}$ is the shifted Heaviside function $H_{i}(x)=H\left(x-x_{i}\right), i=1,2$ and $\langle\cdot, \cdot\rangle$ is the usual $L_{2}$ inner product.

For the same configuration, when the beam is under deformation (bending), the generated charges in terms of voltage across the piezoelectric sensors has the expression (see [DIG])

$$
K_{s} \int_{x_{1}}^{x_{2}} \frac{\partial^{2} y}{\partial x^{2}}(t, x) d x=K_{s}\left(\frac{\partial y}{\partial x}\left(t, x_{2}\right)-\frac{\partial y}{\partial x}\left(t, x_{1}\right)\right)
$$

where $K_{s}$ is a sensor constant which is also a piezoelectric material properties and geometry related quantity.

The system (4) is a formal representation of the dynamics of a damped beam with piezoelectric actuators. To develop computational techniques (e.g., finite elements) based on rigorous convergence arguments, it is necessary to first have a precise formulation of this system.

We start with an abstract formulation. Let $V$ and $H$ be complex Hilbert spaces satisfying $V \hookrightarrow H=H^{*} \hookrightarrow V^{*}$ (see [W] for the construction of this Gelfand triple), where we denote their topological duals by $V^{*}$ and $H^{*}$, respectively. Let $Q$ be the admissible parameter set. The general second order system we consider here is given by

$$
\begin{aligned}
& \langle\ddot{y}(t), \psi\rangle_{V^{*}, V}+\sigma_{1}(q)(y(t), \psi)+\sigma_{2}(q)(\dot{y}(t), \psi)=\langle f(t), \psi\rangle_{V^{*}, V} \quad \text { for } \psi \in V, \\
& y(0)=y_{0}, \quad \dot{y}(0)=y_{1} .
\end{aligned}
$$

Here we use $\langle\cdot, \cdot\rangle_{V^{*}, V}$ to denote the usual [W] duality product. The term $\sigma_{1}(q)$ and $\sigma_{2}(q)$ are parameter dependent sesquilinear forms on $V$ satisfying $V$-ellipticity and 
$V$-continuity conditions. That is, we assume that $\sigma_{1}(q)$ and $\sigma_{2}(q)$ satisfy

$$
\begin{aligned}
& \operatorname{Re} \sigma_{i}(q)(\phi, \phi) \geq k_{i}|\phi|_{V}, \\
& \left|\sigma_{i}(\phi, \psi)\right| \leq c_{i}|\phi|_{V} \cdot|\psi|_{V}, \quad i=1,2
\end{aligned}
$$

for $k_{i}, c_{i}>0, \phi, \psi \in V$. Under weak assumptions on $f$, the system (6) has a unique solution.

Theorem 1 If the sesquilinear forms $\sigma_{1}$ and $\sigma_{2}$ satisfy conditions (7) and (8) with $\sigma_{1}$ symmetric and $f \in L_{2}\left((0, T), V^{*}\right)$, then, for each $w_{0}=\left(y_{0}, y_{1}\right) \in \mathcal{H}=V \times H$, the initial value problem (6) has a unique solution $w(t)=(y(t), \dot{y}(t)) \in L_{2}((0, T), V \times$ $V)$. Moreover, this solution depends continuously on $f$ and $w_{0}$ in the sense that the mapping $\left\{w_{0}, f\right\} \rightarrow w=(y, \dot{y})$ is continuous from $\mathcal{H} \times L_{2}\left((0, T), V^{*}\right)$ to $L_{2}((0, T), V \times$ $V)$.

For a detailed proof of this theorem see [BIW].

Returning to our beam problem, we define the sesquilinear forms by

$$
\begin{aligned}
& \sigma_{1}(q)(y, \phi)=\left\langle E I y^{\prime \prime}, \phi^{\prime \prime}\right\rangle_{L_{2}} \\
& \sigma_{2}(q)(\dot{y}, \phi)=\left\langle c_{D} I \dot{y}^{\prime \prime}, \phi^{\prime \prime}\right\rangle_{L_{2}}+\langle\gamma \dot{y}, \phi\rangle_{L_{2}}
\end{aligned}
$$

with the spaces defined by $V=H_{L}^{2}(0, \ell)=\left\{\phi \in H^{2}(0, \ell) \mid \phi(0)=\phi^{\prime}(0)=0\right\}$ and $H=L_{2}(0, \ell)$ with weighted inner product $\langle\cdot, \cdot\rangle_{H}=\langle\rho \cdot, \cdot\rangle_{L_{2}}$. We assume throughout that $\rho \in L_{\infty}(0, \ell)$ with $\rho(x) \geq \alpha$ for some $\alpha>0$. The term $f(t)=f(t, x)$ is given by

$$
f(t, x)=\frac{1}{\rho} K_{B}\left(H^{\prime \prime}\left(x-x_{2}\right)-H^{\prime \prime}\left(x-x_{1}\right)\right) u(t)+\frac{1}{\rho} \tilde{f}(t, x), \quad 0 \leq x, x_{1}, x_{2} \leq \ell
$$

where $f(t, x)$ belongs to the dual space (see [W]) $V^{*}=\left(H_{L}^{2}(0, \ell)\right)^{*}$ (we assume that $\tilde{f}(t, x) \in V^{*}$ which is a weak assumption on $\left.\tilde{f}\right)$. If both $|E I(\cdot)|_{L_{\infty}} \geq \alpha$ and $\left|c_{D} I(\cdot)\right|_{L_{\infty}} \geq \alpha$ for some $\alpha>0$, then $\sigma_{1}$ and $\sigma_{2}$ are $V$-elliptic and continuous with $\sigma_{1}$ symmetric; hence by Theorem 1 our beam equation is well posed for $f(t, x)$ given by (11).

The parameter estimation problems can be stated in terms of finding parameters which give the best fit of the parameter dependent solutions of the partial differential equations to the observation data for response of the system to various excitations. In our case, the parameters to be estimated include beam mass density $\rho(x)$, stiffness coefficient $E I(x)$ as well as damping parameters $c_{D} I(x), \gamma$ and piezoelectric material parameters $K_{B}, K_{s}$. Let the collection of unknown parameters be denoted by $q=\left(\rho(x), E I(x), c_{D} I(x), \gamma, K_{B}, K_{s}\right)$. For given observations $\left\{z_{i}\right\}$ corresponding to measurements at times $t_{i}$ as obtained in most practical cases, we 
consider the least squares estimation problem of minimizing over $q \in Q$ the least squares functional

$$
J(y, z ; q)=\left|\tilde{C}_{2}\left(\tilde{C}_{1}\left\{y\left(t_{i}, \cdot ; q\right)\right\}-\left\{z_{i}\right\}\right)\right|^{2}
$$

where $\left\{y\left(t_{i}, \cdot ; q\right)\right\}$ are the parameter dependent weak solutions of (4) or (6) evaluated at each time $t_{i}, i=1,2, \cdots, \bar{N}$ and $|\cdot|$ is an appropriately chosen Euclidian norm. The set $Q$ is some admissible parameter set. The operator $\tilde{C}_{1}$ may have several forms depending on the type of sensors being used. When the collected data are displacement, velocity, or acceleration at a point $\bar{x}$ on the beam, we minimize

$$
\left.J_{\nu}(q)=\sum_{i=1}^{\bar{N}} \mid \frac{\partial^{\nu} y}{\partial t^{\nu}}\left(t_{i}, \bar{x} ; q\right)-z_{i}\right)\left.\right|^{2}
$$

for $\nu=0,1,2$, respectively. In this case the operator $\tilde{C}_{1}$ involves differentiation (either $\nu=0,1$ or 2 times, respectively) with respect to time followed by pointwise evaluation in $t$ and $x$. When a piezoelectric sensor is used, the functional to be minimized is

$$
\left.J_{p}(q)=\sum_{i=1}^{\bar{N}} \mid K_{s}\left(\frac{\partial y}{\partial x}\left(t_{i}, x_{2} ; q\right)-\frac{\partial y}{\partial x}\left(t_{i}, x_{1} ; q\right)\right)-z_{i}\right)\left.\right|^{2},
$$

for the piezoelectric elements being located on the beam between $x_{1}$ and $x_{2}$. Here $\left\{z_{i}\right\}$ are the measured voltages across the piezoelectric elements.

The operator $\tilde{C}_{2}$ may be the identity (corresponding to time domain identification procedures as in (13) and (14)) or the Fourier transform (corresponding to identification in the frequency domain). If the identification is carried out in the frequency domain and the operator $\tilde{C}_{2}$ is a Fourier transform operator, the corresponding cost functional is given by

$$
\hat{J}(q)=\sum_{\ell=1}^{\bar{N}_{M}}\left(\epsilon_{1}\left|\mathrm{f}_{k_{\ell}^{y}}(q)-\mathrm{f}_{k_{\ell}}\right|^{2}+\epsilon_{2} \sum_{j=-n_{\ell}}^{N_{\ell}}|| U\left(k_{\ell}^{y}+j ; q\right)|-| Z\left(k_{\ell}^{z}+j\right)||^{2}\right),
$$

where $U(k ; q)$ and $Z(k)$ are the Fourier series coefficients of $\tilde{C}_{1}\left\{y\left(t_{i}, \bar{x} ; q\right)\right\}$ and $\left\{z_{i}\right\}$ respectively, $\mathrm{f}_{k_{\ell}^{y}}$ and $\mathrm{f}_{k_{\ell}^{z}}$ are the $\left(k_{\ell}^{y}\right)^{t h}$ vibration frequency of the solution $U(k ; q)$ and the $\left(k_{\ell}^{z}\right)^{t h}$ frequency of the observation data $Z(k), \epsilon_{1}, \epsilon_{2}$ are weighting constants, and $n_{\ell}, N_{\ell}$ are certain lower and upper limits associated with the width (or the support) of the $\ell^{t h}$ spikes. In obtaining (15), we have assumed that there are a finite and distinct number $\bar{N}_{M}(<\bar{N})$ of "spikes", i.e. vibration frequencies, among the $Z(k)$ and the number of spikes of the solution $U(k ; q)$ is the same as $\bar{N}_{M}$. We refer to [BW] for a detailed derivation and discussion of the cost functional (15). 
The minimization in our parameter estimation problems involves an infinite dimensional state space and an infinite dimensional admissible parameter set (of functions). We thus consider Galerkin type approximations in the context of the variational formulation of (6). Let $H^{N}$ be a sequence of finite dimensional subspaces of $H$, and $Q^{M}$ be a sequence of finite dimensional sets approximating the parameter set $Q$. We define the orthogonal projections $P^{N}: H \rightarrow H^{N}$ of $H$ onto $H^{N}$. Then a family of approximating estimation problems with finite dimensional state spaces and parameter sets can be formulated by seeking $q \in Q^{M}$ which minimizes

$$
J^{N}\left(y^{N}, z ; q\right)=\left|\tilde{C}_{2}\left(\tilde{C}_{1}\left\{y^{N}\left(t_{i}, \cdot ; q\right)\right\}-\left\{z_{i}\right\}\right)\right|^{2},
$$

where $y^{N}(t ; q) \in H^{N}$ is the solution to the finite dimensional approximation of $(6)$ given by

$$
\begin{aligned}
& \left\langle\ddot{y}^{N}(t), \psi\right\rangle_{V^{*}, V}+\sigma_{1}(q)\left(y^{N}(t), \psi\right)+\sigma_{2}(q)\left(\dot{y}^{N}(t), \psi\right)=\langle f(t), \psi\rangle_{V^{*}, V} \\
& y^{N}(0)=P^{N} y_{0}, \quad \dot{y}^{N}(0)=P^{N} y_{1}
\end{aligned}
$$

for $\psi \in H^{N}$.

For the parameter sets $Q$ and $Q^{M}$, and state spaces $H^{N}$, we make the following hypotheses:

(H1) The sets $Q$ and $Q^{M}$ lie in a metric space $\tilde{Q}$ with metric $d$ with $Q, Q^{M}$ compact in this metric and there is a mapping $i^{M}: Q \rightarrow Q^{M}$ so that $Q^{M}=i^{M}(Q)$. Furthermore, for each $q \in Q, i^{M}(q) \rightarrow q$ in $\tilde{Q}$ with the convergence uniform in $q \in Q$;

(H2) The finite dimensional subspaces $H^{N}$ satisfy $H^{N} \subset V$ as well as the approximation properties: For each $\psi \in V,\left|\psi-P^{N} \psi\right|_{V} \rightarrow 0 \quad$ as $N \rightarrow \infty$.

In addition to (uniform in $Q$ ) ellipticity and continuity conditions (7) and (8), the sesquilinear forms $\sigma_{1}(q)$ and $\sigma_{2}(q)$ are assumed to be defined on $Q$ and satisfy the continuity-with-respect-to-parameter conditions

$$
\left|\sigma_{i}(q)(\phi, \psi)-\sigma_{i}(\tilde{q})(\phi, \psi)\right| \leq \gamma_{i} d(q, \tilde{q})|\phi|_{V}|\psi|_{V}, \quad i=1,2,
$$

for $\phi, \psi \in V$ and $q, \tilde{q} \in Q$ where the constants $\gamma_{1}, \gamma_{2}$ depend only on $Q$.

Solving the approximate estimation problems involving (16) and (17), we obtain a sequence of estimates $\left\{\bar{q}^{N, M}\right\}$. To obtain parameter estimate convergence and continuous dependence (with respect to the observations $\left\{z_{i}\right\}$ ) results, it has been shown in $[\mathrm{B}],[\mathrm{BK} 3]$ and $[\mathrm{BWI}]$ that it is suffices, under the assumption that $Q$ is a compact set, to argue that for arbitrary $\left\{q^{N, M}\right\} \in Q$ with $q^{N, M} \rightarrow q \in Q$, we have

$$
\tilde{C}_{2} \tilde{C}_{1} y^{N}\left(t ; q^{N, M}\right) \rightarrow \tilde{C}_{2} \tilde{C}_{1} y(t ; q)
$$


for each $t$.

The following theorem and corollary establish the convergence (19) for all forms of $\tilde{C}_{1}, \tilde{C}_{2}$ that are of interest to us here.

Theorem 2 Suppose that $Q, Q^{M}$ satisfy (H1) and $H^{N}$ satisfies (H2). Assume that the sesquilinear forms $\sigma_{1}(q)$ and $\sigma_{2}(q)$ satisfy (7), (8), (18). Furthermore, assume that

$$
q \rightarrow f(t ; q) \text { is continuous from } Q \text { to } L_{2}\left((0, T), V^{*}\right) \text {. }
$$

Let $q^{N, M}$ be arbitrary in $Q$ such that $q^{N, M} \rightarrow q$ in $Q$; then for $t>0$ as $N, M \rightarrow \infty$ we have

$$
\frac{\partial^{\nu} y^{N}}{\partial t^{\nu}}\left(t ; q^{N, M}\right) \rightarrow \frac{\partial^{\nu} y}{\partial t^{\nu}}(t ; q) \quad \text { in } V \text { norm, for } \nu=0,1,2,
$$

where $y^{N}, \dot{y}^{N}$ are the solutions to (17) and $y, \dot{y}$ are the solutions to (6).

Furthermore we have

Corollary 2.1 Under the assumptions of Theorem 2, we have as $N, M \rightarrow \infty$

$$
\begin{gathered}
\frac{\partial y^{N}}{\partial x}\left(t ; q^{N, M}\right) \rightarrow \frac{\partial y}{\partial x}(t ; q) \quad \text { in } H^{1} \text { norm, for } t>0, \\
\sum_{\ell=1}^{\bar{N}_{M}}\left(\epsilon_{1}\left|f_{k_{\ell}^{y^{N}}}\left(q^{N, M}\right)-f_{k_{\ell}^{y}(q)}\right|^{2}+\epsilon_{2} \sum_{j=-n_{\ell}}^{N_{\ell}}|| U^{N}\left(k_{\ell}^{y^{N}}+j ; q^{N, M}\right)|-| U\left(k_{\ell}^{y}+j ; q\right)||^{2}\right) \rightarrow 0,
\end{gathered}
$$
where $U^{N}\left(k ; q^{N, M}\right)$ and $U(k ; q)$ are the Fourier series coefficients for $\tilde{C}_{1}\left\{y^{N}\left(t ; q^{N, M}\right)\right\}$ and $\tilde{C}_{1}\{y(t ; q)\}$, respectively.

The corollary follows from Theorem 2. For a detailed proof when $\tilde{C}_{2}$ is the Fourier transform see [BWI]. For the proof of Theorem 2, we refer the reader to [BWIS] for the cases $\nu=0,1$ and $[\mathrm{BR}]$ for the case $\nu=2$.

We have outlined a framework for methods to carry out parameter identification for distributed parameter systems in both the time domain and the frequency domain. The motivation for introducing a frequency domain technique is that the time domain techniques give poor results when the observations contain several vibration modes and the initial guesses for parameters are not close to the optimal ones ([WA]). In such a situation we use a hybrid of these two methods: we iterate in a frequency domain formulation to obtain a reasonable initial parameter estimate for the time domain formulation which is then used to complete the identification procedure.

In Theorem 2, we presented convergence results allowing approximation of the parameter set. This form of the theoretical foundation provides a sound necessary basis for the detection and quantification of damage in structures since damage affects 
the dynamic behavior of systems in terms of changes in the system parameters such as $\rho$ and $I$.

In concluding this section we make further comments related to the various allowable forms of the operator $\tilde{C}_{1}$. The flexibility in the choice of $\tilde{C}_{1}$ and hence flexibility in the choice of sensors in the above theoretical framework makes it possible to carry out cross experiments to test the reliability of our methods. Even more important, this flexibility in our method enables us to use smart materials to carry out damage detection by employing only piezoelectric materials in actuating and sensing for the structure.

\section{Quantitative and Experimental Results}

In implementing the computational method outlined in Section 2, we chose cubic splines as the basis elements. The finite dimensional space $H^{N}$ is defined as the span of the basis set for $S_{3, B}^{N}(0, \ell)$ (the cubic B-splines corresponding to mesh size $\ell / N$ - see $[\mathrm{P}]$ ) with the basis set modified to satisfy the essential boundary conditions $\phi(0)=\phi^{\prime}(0)=0$. The finite dimensional space $H^{N}$ constructed in this manner satisfies hypothesis (H2) (for details see [BWIS]). The dimension of the resulting approximation space is $N+1$. For our calculations we chose $N=10$, since the eigenvalues of this approximate finite dimensional system become stable at and after $N=10$ in the sense that the eigenvalues did not change significantly as $N$ increases beyond 10 .

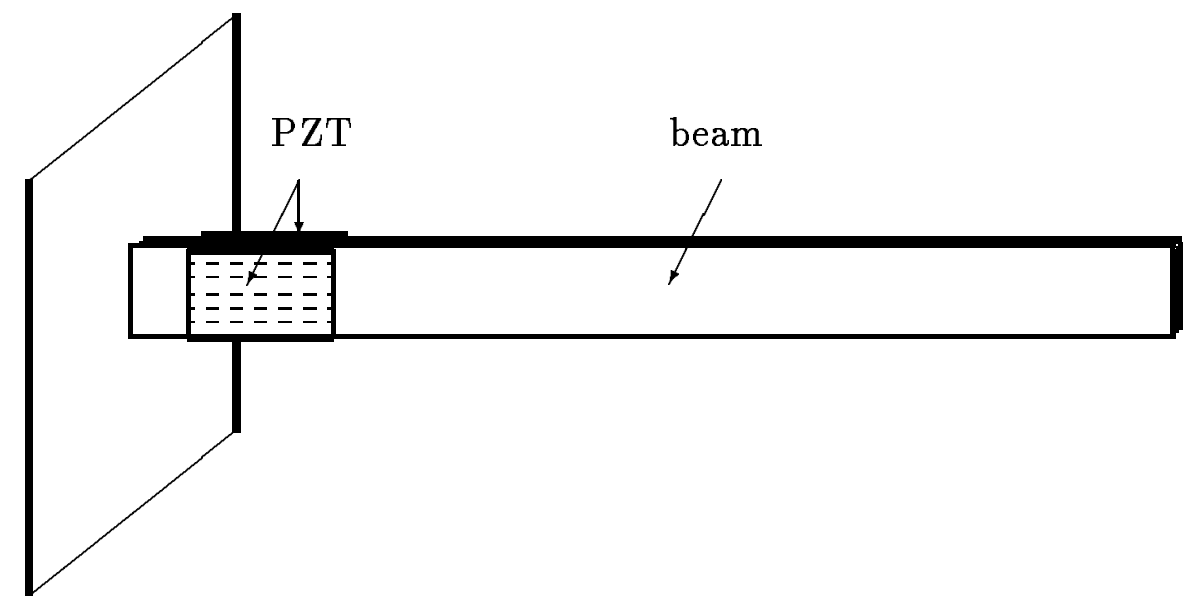

Figure 3.1: Test beam with piezoceramic patches.

In the numerical examples we present next, an undamaged beam with two 


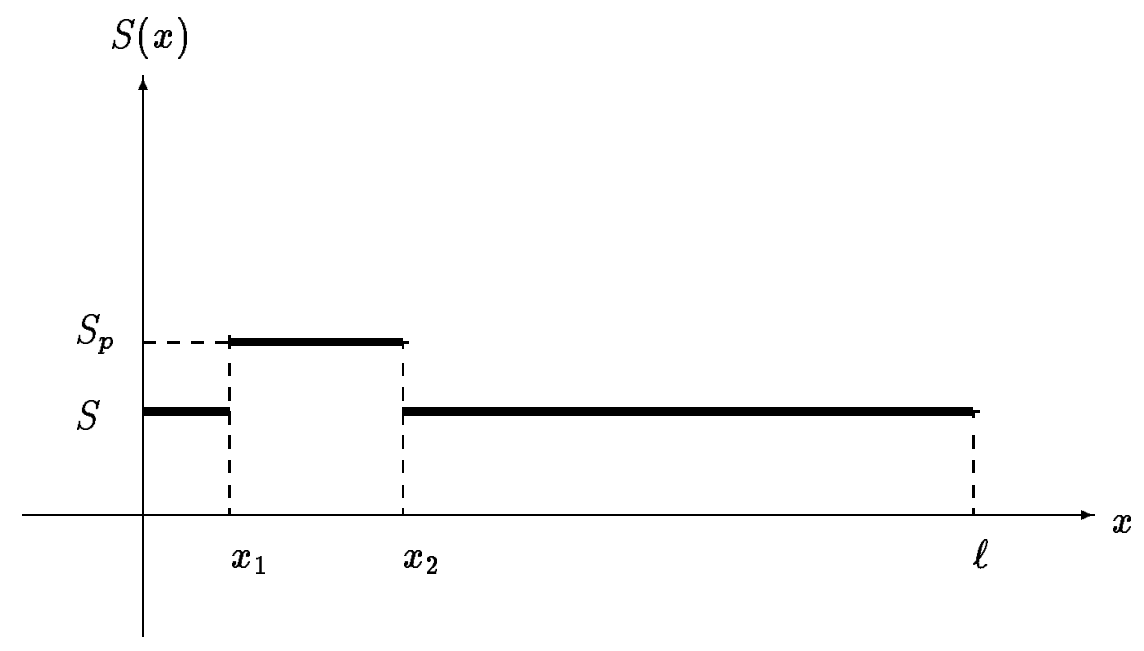

Figure 3.2: $\rho(x), E I(x), c_{D} I(x)$ function shape $S(x)$.

attached piezoceramic patches (PZT) as shown in Figure 3.1 was used as a test structure to demonstrate the consistency of identification results for our computational method across different excitation and sensing mechanisms. To accurately model the geometry of the structure, we assumed that the mass density, stiffness and KelvinVoigt damping coefficients are piecewise constant functions as depicted in Figure 3.2. These assumptions were subsequently verified to be most reasonable. For the choice of parameters $q=\left(\rho(x), E I(x), c_{D} I(x), \gamma, K_{B}, K_{s}\right)$, we take the parameter space $\tilde{Q}$ to be $\left[L_{\infty}(0, \ell)\right]^{3} \times \mathbb{R}^{3}$, and the parameter set $Q$ to be a uniformly bounded collection of piecewise constant functions each having jump discontinuities at most at $x_{1}$ and $x_{2}$. If we choose the mapping $i^{M}$ as the identity, then hypothesis (H1) is satisfied.

Experiments with a test beam as depicted in Figure 3.1 were carried out at the Mechanical Systems Laboratory, then located at the State University of New York at Buffalo (now at Virginia Polytechnic Institute and State University). The measured dimensions of the actual structure are given in Table 3.1 and the handbook values for the physical characteristics of this aluminum (2024-T4) beam/piezo (G-1195 PZT ceramic) structure are given in Table 3.2. In the tables the subscripts indicate the materials: $b$ for beam and $p$ for piezoceramic, $\ell$ is length, $w$ is width, $t$ is thickness, $E$ is the Young's modulus, and $\rho$ is the mass density. The patches were bonded to the beam on the opposite sides of the beam at the same position.

The beam was clamped at $x=0$. The piezoceramic patches were placed between $x_{1}=2.54 \mathrm{~cm}$ and $x_{2}=8.89 \mathrm{~cm}$ (or $1^{\prime \prime}$ and $3.5^{\prime \prime}$ respectively) on the beam. The time response data and input signal from the experimental beam were obtained 


\begin{tabular}{|c|c|c|c|c|c|c|}
\hline Unit & $\ell_{b}$ & $w_{b}$ & $t_{b}$ & $\ell_{p}$ & $w_{p}$ & $t_{p}$ \\
\hline Metric & $45.73 \mathrm{~cm}$ & $2.03 \mathrm{~cm}$ & $0.16 \mathrm{~cm}$ & $6.37 \mathrm{~cm}$ & $2.03 \mathrm{~cm}$ & $0.0254 \mathrm{~cm}$ \\
\hline English & $18^{\prime \prime}$ & $0.8^{\prime \prime}$ & $.0625^{\prime \prime}$ & $2.5^{\prime \prime}$ & $0.8^{\prime \prime}$ & $0.01^{\prime \prime}$ \\
\hline
\end{tabular}

Table 3.1: Dimension of the beam and piezoceramic patch.

\begin{tabular}{|c|c|c|c|c|}
\hline Unit & $E_{b}$ & $\rho_{b}$ & $E_{p}$ & $\rho_{p}$ \\
\hline Metric & $7.3 \times 10^{6} \mathrm{~N} / \mathrm{cm}^{2}$ & $2.766 \mathrm{~g} / \mathrm{cm}^{3}$ & $6.3 \times 10^{6} \mathrm{~N} / \mathrm{cm}^{2}$ & $7.6 \mathrm{~g} / \mathrm{cm}^{3}$ \\
\hline English & $10.6 \times 10^{6} \mathrm{psi}$ & $0.1 \mathrm{\ell b} / \mathrm{in}^{3}$ & $9.148 \times 10^{6} \mathrm{psi}$ & $0.275 \mathrm{\ell b} / \mathrm{in}^{3}$ \\
\hline
\end{tabular}

Table 3.2: Characteristics of the beam and piezoceramic patch.

using a Tektronix Analyzer (model 2600). For more detailed descriptions of the experimental setup and the parameter identification procedure, see [BWIS].

In examining the ability of our method to estimate parameters reliably, independent of the type of excitation or sensing device used, two separate experiments were performed. In the first experiment an impulse hammer was used as the input device and piezoceramic patches were used as a sensor. In the second experiment, the excitation was obtained via an input voltage to the patches and observation data was recorded from an accelerometer. At a sampling frequency of $256 \mathrm{~Hz}$ for $16 \mathrm{sec}-$ onds, two modes (at $6.625 \mathrm{~Hz}$ and $38.375 \mathrm{~Hz}$ ) were observed in the response in each experiment.

The results (graphs comparing the model response after ID of parameters with the experimental data) for the first identification experiment are given in Figure 3.3.

In this example, the beam was excited by an impulse force applied (via the impulse hammer) to the beam along the neutral axis at $2.54 \mathrm{~cm}\left(1^{\prime \prime}\right)$ away from the clamped end. Then the forcing function in (11) becomes (since $u \equiv 0$ )

$$
f(t, x)=\tilde{f}(t, x)
$$

with $\tilde{f}$ given by the impulse. The recorded signal exhibited a triangular shape. The voltages across the patches due to the beam vibrations were collected as observation data. 